\title{
Trivium
}

Revue franco-allemande de sciences humaines et sociales - Deutsch-französische Zeitschrift für Geistesund Sozialwissenschaften

$10 \mid 2012$

Lisibilité

\section{Le Rituel du Serpent d'Aby Warburg. Correspondances entre la lecture de textes culturels et de textes écrits}

\section{Sigrid Weigel}

Traducteur : Cécile Chamayou-Kuhn

\section{OpenEdition}

\section{Journals}

Édition électronique

URL : http://journals.openedition.org/trivium/4125

DOI : $10.4000 /$ trivium. 4125

ISSN : 1963-1820

\section{Éditeur}

Les éditions de la Maison des sciences de l'Homme

\section{Référence électronique}

Sigrid Weigel, « Le Rituel du Serpent d'Aby Warburg. Correspondances entre la lecture de textes culturels et de textes écrits », Trivium [En ligne], 10 | 2012, mis en ligne le 30 mars 2012, consulté le 10 décembre 2020. URL : http://journals.openedition.org/trivium/4125 ; DOI : https://doi.org/10.4000/ trivium. 4125

Ce document a été généré automatiquement le 10 décembre 2020.

\section{(c) (i) () $\Theta$}

Les contenus des la revue Trivium sont mis à disposition selon les termes de la Licence Creative Commons Attribution - Pas d'Utilisation Commerciale - Pas de Modification 4.0 International. 


\title{
Le Rituel du Serpent d'Aby Warburg. Correspondances entre la lecture de textes culturels et de textes écrits ${ }^{1}$
}

\author{
Sigrid Weigel \\ Traduction : Cécile Chamayou-Kuhn
}

\section{NOTE DE L'ÉDITEUR}

Nous remercions Sigrid Weigel de nous avoir accordé l'autorisation de traduire ce texte pour le présent numéro.

1 La lecture du Rituel du Serpent d'Aby Warburg soulève des questions d'ordre philologique portant sur la lisibilité de ce texte. Elles ouvrent des perspectives ethnologiques qui abordent la question de la lisibilité des signes propres à une culture étrangère. Cette tentative d'Aby Warburg, qui consistait à comprendre le système symbolique des Indiens Pueblos ou encore à lire une culture qui lui était étrangère à la manière d'un texte culturel, s'apparente très nettement au phénomène des images textuelles qui se forment en nous à propos de cultures étrangères en lisant des récits de voyages ethnologiques ou littéraires. Aussi Le Rituel du Serpent d'Aby Warbug me paraîtil particulièrement pertinent pour ouvrir une discussion sur les aspects que recouvre la notion de lecture, et ce en l'abordant à travers le prisme d'une anthropologie culturelle. À l'intersection entre, d'une part, la thématique de la lisibilité de ce livre publié en 1988 sous le titre Le Rituel du Serpent [Schlangenritual] et, d'autre part, les questions que Warburg y pose à propos de la lisibilité d'une culture étrangère, c'est-àdire à partir des correspondances entre la lisibilité d'un texte littéraire et celle d'un texte culturel, il s'agira ici de développer, à l'aide d'exemples, les possibilités et les perspectives méthodologiques d'un paradigme propre à une anthropologie culturelle. En ce sens, il sera question des traces de paradigmes appartenant aux sciences de la culture dans la lecture d'un texte ou, vice versa, il sera question des possibilités et des 
difficultés inhérentes à une approche anthropologique du point de vue des études littéraires, c'est-à-dire d'une discipline autonome et issue de la philologie.

\section{L'ancrage scientifique d'un livre hybride}

2 Le Rituel du Serpent s'impose comme l'objet d'une telle discussion pour la bonne et simple raison que ce texte, qui fut publié pour la première fois en 1988 en allemand, nous place devant des difficultés considérables. Or, celles-ci ne peuvent être résolues, ni même abordées sous l'angle d'une seule discipline. Si le nom de l'auteur semble faire appel au savoir des historiens de l'art, sous l'égide desquels Warburg a jusqu'à présent joué un rôle en tant que fondateur d'une école iconographique, le sous-titre de l'ouvrage - « un récit de voyage » - vient mettre ceci en doute, pour faire entrer en scène les littéraires et éventuellement les historiens dont on sait qu'ils se sont consacrés à l'étude de documents provenant de l'histoire de la littérature de voyage. Pour peu qu'ils ouvrent le livre et découvrent le titre bien différent qui figure sur la page de titre: «Images du territoire des Pueblos en Amérique du Nord»,beaucoup d'entre eux voudront alors confier ce livre aux ethnologues. Il est possible que ceux-ci, à leur tour, ne soient pas véritablement enchantés, tout au moins pas au deuxième coup d'œil lorsqu'ils trouveront sur la troisième page un autre titre signalant que cette publication est un texte écrit dans une clinique psychiatrique : « conférence prononcée à la clinique Bellevue à Kreuzlingen le 21 avril $1923 \%$. Ce texte étant ainsi passé de mains en mains, je ne saurais dire si les psychologues seront encore prêts à endosser la responsabilité d'un hommage et d'une interprétation. La situation, telle qu'elle vient d'être décrite, aura peut-être conduit à un litige quant aux domaines de compétences qu'engagent les écrits de Warburg. Et les pages suivantes du livre n'apporteront aucune précision à ce sujet: elles laissent en effet apparaitre que le texte est accompagné d'images dont une partie pourrait effectivement provenir d'archives ethnographiques. L'autre partie, en revanche, est constituée de manuscrits médiévaux et de sculptures antiques.

3 Le Rituel du Serpent étant à l'origine une conférence rédigée durant le séjour de Warburg au sanatorium de Ludwig Binswanger, on notera en outre qu'il existe des liens entre la Suisse et Hambourg où Warburg avait fondé en 1909 sa bibliothèque, la Kulturwissenschaftliche Bibliothek Warburg. C'est en effet à Hambourg que Warburg sombra dans la maladie en 1918; une maladie qui devait trouver son origine, ainsi qu'on le relate, dans son environnement culturel, dans la violence qui lui était inhérente et qui avait fait surface durant la Première Guerre mondiale. C'est en Suisse qu'il a recouvré la santé. Dans son esprit pourtant, il a séjourné dans un autre lieu ; un troisième lieu: dans les images-souvenirs d'un voyage en Amérique du Nord durant l'année 1895 ; un voyage effectué 27 ans avant la conférence qui l'avait conduit vers les territoires indiens du Nouveau-Mexique et de l'Arizona. De même, il a séjourné dans les archives de sa propre mémoire iconographique ; laquelle contenait tant les œuvres de la Renaissance italienne et les formules imagées antiques qui y sont citées, que des images empruntées à l'histoire de l'astrologie.

Si je me consacre ici à ce livre hybride publié sous le titre du Rituel du Serpent, ce n'est pas pour traiter de quelque perspective disciplinaire tirée de la palette précédemment évoquée - même si l'addition d'aspects relevant de disciplines isolées est effectivement courante et porte le nom d'interdisciplinarité, notamment lorsqu'il s'agit de convoquer 
les mécènes. Il s'agira plutôt de nous concentrer sur les points où des perspectives divergentes se croisent, entrent éventuellement en conflit ou ouvrent sur un autre paradigme méthodique. En référence aux évolutions actuelles de la théorie, telles qu'elles apparaissent dans les domaines anglo-saxons et français, je nommerai provisoirement ce paradigme "culture anthropologique». Il représente un champ d'études incluant des questions relatives à la lisibilité de textes écrits, mais aussi à ce que l'on appelle des textes culturels, qu'il s'agisse de narrations, de gestes, de pratiques ou d'objets, de topographies ou encore de ces restes et de ces traces dont le déchiffrage est primordial pour tous ceux qui cherchent à interpréter les cultures passées.

Il s'agira donc aussi de la question de savoir si les recherches contemporaines effectuées au sein de ce champ peuvent être rattachées à Aby Warburg, dont les travaux n'ont eu de cesse de changer d'ancrage terminologique: il les nomma par exemple psychologie historique de l'expression humaine, ou formules originelles de la langue des signes. Il les rangea dans la catégorie de la psychologie de l'art, de l'histoire des cultures, de l'histoire des religions, de l'ethnographie, ou les concevait comme une contribution à la mémoire sociale. Prenons son concept le plus célèbre, la « formule du pathos» [Pathosformel], par lequel Warburg désigne les gestes représentés dans les tableaux et autres médias visuels qu'il considère comme des symboles mémoriels d'angoisses et d'émotions dites dépassées. Ce concept met en corrélation des aspects empruntés à l'histoire de l'art, au langage corporel (c'est-à-dire à un knowledge incorporated), à la mémoire culturelle ou à l'imaginaire collectif. Lorsque Warburg étudie les «formules du pathos » sous l'angle de la formation de symboles et qu'il les conçoit, dans une perspective anthropologique, comme une fonction de l'activité civilisationnelle, ses études acquièrent une dimension appartenant à ce que l'on qualifierait aujourd'hui de sémiologie culturelle. Ses travaux s'inscrivent également dans un cadre contemporain axé autour de l'histoire culturelle de l'Europe, dans laquelle les grandes lignes tracées par les Lumières sont surtout abordées à rebours. Le leitmotiv des études de Warburg est en effet constitué par la question qu'il pose au sujet des formes de «survivance» [Nachleben] de l'Antiquité dans la culture de la Renaissance et, plus particulièrement, sur le côté démoniaque, sauvage ou - ainsi que le formule Warburg - sur le côté «païen » de cette Antiquité. Et c'est précisément dans son « origine sauvage ${ }^{2}$ » que réside actuellement pour l'anthropologie de l'Antiquité un intérêt évident.

6 La publication du Rituel du Serpent en 1988 doit être replacée dans le contexte d'une sorte de redécouverte de Warburg qui s'est manifestée par de nombreuses publications, des colloques ainsi qu'une exposition à Vienne en 1993 : après la redécouverte de pièces disparues depuis longtemps, sa " collection d'images sur l'histoire de l'astrologie et de l'astronomie » [Bildersammlung zur Geschichte von Sternglaube und Sternkunde] (1930) y fut en effet reconstituée. De même, les planches de son atlas d'images Mnémosyne y furent exposées pour la première fois dans de telles proportions ${ }^{3}$. C'est surtout l'atlas d'images qui, parce qu'il présente sa collection de gestes dans les domaines de l'art et d'autres médias visuels sous l'aspect d'une mémoire sociale, a contribué à ce que le nom de Warburg soit aujourd'hui encore connu, et ce au-delà des frontières de l'histoire de l'art. Car c'est précisément - tout au moins dans la sphère germanophone - l'étude de l'histoire et de la théorie de la mémoire qui suscite aujourd'hui à la fois nombre de coopérations interdisciplinaires et le développement de méthodes propres aux sciences de la culture. Parallèlement, Le Rituel du Serpent a accru l'attrait international que pouvait exercer Warburg. Il se peut que cela soit dû au fait que la problématique d'une 
continuité entre l'Antiquité et la Renaissance (problématique relevant d'un paradigme plutôt conventionnel) y soit déplacée dans une optique ethnologique vers la question du rapport qu'entretiennent la pensée sauvage et la modernité. Et parce que d'autres disciplines ont adopté le regard ethnologique, à savoir l'étude d'une culture passée ou d'une culture propre en tant que culture étrangère - ce qui peut être conçu comme l'origine des débats contemporains sur la culture anthropologique - Warburg est devenu un centre d'intérêt majeur.

\section{Warburg : un anthropologue culturel. Apories et césures}

Dans son essai intitulé "Aby Warburg as Historical Anthropologist », Peter Burke a qualifié le voyage effectué par Warburg durant près de trois mois dans différentes colonies pueblos de recherche de terrain ${ }^{4}$. Il a en outre comparé sa méthode aux évolutions actuelles, telles qu'elles apparaissent au sein de l'histoire des mentalités ou encore de la micro-histoire :

« Je souhaiterais souligner que l'intérêt que portait Warburg à d'autres cultures l'a

aidé à comprendre non seulement l'Antiquité classique, mais aussi la

Renaissance ${ }^{5}$. »

Et bien que Burke soit l'une des rares personnes qui aient attiré l'attention sur la nécessité de replacer la terminologie de Warburg dans son époque (car elle demeure problématique en raison des postulats évolutionnistes et de la méconnaissance d'autres cultures qui y est liée), sa tentative de réactualisation - ne fût-ce qu'en conclusion de son essai - permet de dépasser de considérables clivages entre la pensée de Warburg et les débats actuels. Mais lorsqu'il est précisément question d'une approche spécifique consistant à accentuer le caractère étranger du passé, il conviendrait aussi et avant tout d'attribuer cette approche à Warburg lui-même, il conviendrait de faire du trouble et de la surprise que génère la lecture de ses textes le point de départ d'une analyse de sa pensée. En somme, il conviendrait de réfléchir aux différences et aux césures qui creusent un abîme entre notre pratique académique et des recherches effectuées dans la Kulturwissenschaftliche Bibliothek Warburg. Dans les faits néanmoins, la réception de Warburg obéit à une sorte de reterritorialisation à l'intérieur d'un périmètre qu'il avait pourtant quitté. C'est ce qui transparait dans le souhait de réimplanter ses travaux dans une discipline ou dans les efforts entrepris pour réinstaller la Kulturwissenschaftliche Bibliothek Warburg à Hambourg après ses nombreux exils.

Il importe tout d'abord de mentionner l'aversion de Warburg pour l'organisation du savoir selon des disciplines et des époques strictement délimitées; aversion bien connue de ses contemporains et que l'on aime à citer en reprenant sa célèbre formule : « des frontières policières ${ }^{6}$ ». Dans son essai sur Bachofen (1935), Walter Benjamin place Warburg au même niveau que Goethe et Bachhofen, invoquant «le mépris pour les limites établies entre les sciences ${ }^{7}$ ». Contrairement à Benjamin, qui ne choisit pas de travailler à l'extérieur de l'université, il semble que Warburg n'ait jamais prétendu à une fonction académique, et ce même lorsque en 1918 l'accès n'en fut plus interdit aux Juifs non baptisés d'Allemagne. Soutenu par son propre institut de recherches indépendant, la Kulturwissenschaftliche Bibliothek, il s'engagea plutôt pour la création d'une université qui manquait encore à la ville de Hambourg au début du siècle (et dont la fondation devait attendre jusqu'en 1919, la ville hanséatique s'étant en effet d'abord 
décidée pour la création d'un institut colonial). Toutefois, après qu'une coopération intensive avec certains chercheurs de la nouvelle université se fut développée, en l'occurrence avec Ernst Cassirer et Erwin Panofsky, la bibliothèque dut être transférée en 1933 à Londres en raison de l'arrivée au pouvoir des national-socialistes.

Sur cette toile de fond, la notion de "retour chez soi " [Heimkehr], dont s'est servi récemment Salvatore Settis pour intituler son plaidoyer en faveur de la reconstruction de la Kulturwissenschaftliche Bibliothek Warburg à Hambourg, contient une double erreur. Le souhait de " combler le vide » existant depuis 1933 et de "panser la plaie ${ }^{8}$ ", au lieu de les considérer comme un symptôme, c'est-à-dire comme le symbole mémoriel d'une histoire des sciences allemandes, ne permet effectivement pas de voir qu'un retour de l'exil ne saurait correspondre à un retour chez soi. De plus, la notion de « retour chez soi » semble suggérer que ce « chez soi » préexiste à l'exil.

«Le National-Socialisme et la persécution des Juifs ont à jamais arraché la Kulturwissenschaftliche Bibliothek Warburg à son environnement naturel, à savoir à la culture allemande qui l'avait pourtant vu naître 9 ."

Une telle représentation des choses fait à nouveau surgir le mythe d'une symbiose entre Juifs et Allemands qui aurait simplement été interrompue ou, plus précisément, qui aurait été taxée de symbiose contre-nature par les nazis. Parallèlement, il ne s'agit pas non plus d'un hasard si les métaphores et la terminologie sur lesquelles cette représentation est bâtie, en l'occurrence l'association d'une culture nationale à un fait naturel et à un berceau, se rattachent à une approche anthropologique obsolète en raison de ses fondements biologiques et pour laquelle le nazisme est devenu une sorte d'épreuve de vérité raciale.

10 Car pour l'histoire scientifique de l'anthropologie qui s'est développée au tournant du siècle avec la découverte des origines "primitives » de l'Antiquité et sous l'impulsion de l'archéologie, de l'ethnologie, de l'histoire comparée des religions ainsi que de la philologie, l'année 1933 marque une césure : les discours nationalistes ou apologétiques chrétiens latents se transformèrent effectivement en un antisémitisme manifeste. Dans une étude portant sur le champ - en soi extrêmement ambivalent - de l'anthropologie, telle qu'elle est pratiquée dans le sillage de Hermann Usener, le mentor d'Aby Warburg, Renate Schlesier s'est penchée sur le spectre hétérogène des « ouvriers dans le vignoble de Usener». Elle a par ailleurs attiré l'attention sur la dépendance de "beaucoup d'archéologues allemands » à l'égard des idées nationalistes ${ }^{10}$.

11 En ce qui concerne Usener lui-même - et son élève Warburg a abondé dans son sens à cet égard -, il s'agit plutôt d'une tendance aux explications universelles, qui s'attache aux mythes des origines et qui, cultivant une approche comparative entre les références antiques et ethnologiques, efface leurs différences. Parce que Warburg s'appuie dans ses textes sur la catégorie d'humanité, ainsi que sur les "grands processus de développement généraux ${ }^{11}$ » et qu'il décrit un système global de formes symboliques ${ }^{12}$ à l'aide d'une typologie des seuils de développement, cette tendance à l'universalisation fait figure de garde-fou qu'il doit opposer au dépassement des catégories propres aux disciplines et aux époques. Néanmoins, l'histoire de la culture menace à nouveau de se présenter comme une histoire naturelle en raison de cette tendance.

De fait, dès lors que l'humanité de l'être humain est mise en exergue pour constituer une histoire de la culture, et ce par le recours au binarisme " nature-culture ", l'histoire de l'Homme revêt à nouveau les traits d'une histoire naturelle. Car elle est alors abordée selon une perspective universaliste défendant la théorie d'une essence de 
l'Homme. Afin de rompre avec cette aporie propre à toute approche anthropologique, le débat théorique actuel se fonde sur une reformulation, elle-même ancrée dans les champs d'une théorie de la différence ainsi que d'une sémiotique culturelle, en un mot, dans une anthropologie culturelle. Ainsi est-ce le problème de la lisibilité, c'est-à-dire de la lecture qui est placé au centre, et non plus la question concernant des explications exhaustives ou une évolution générale.

La référence la plus connue dans ce contexte est sans doute la « description dense » de Clifford Geertz ${ }^{13}$, c'est-à-dire son concept de lisibilité qui trouve ses racines dans la situation de perception d'un ethnographe à qui fait défaut non seulement le code de traduction, mais aussi les clefs lui permettant de savoir si un geste peut être décrypté comme une parole involontaire, comme un signe ou comme une parodie. De fait, cette description exige une posture analytique qui s'appuie sur des expériences faites dans le contexte de langages symboliques et de textes. Lorsque Geertz compare cette posture à la tentative "de lire (au sens de « construire une lecture de ») un manuscrit étranger, défraîchi, plein d'ellipses, d'incohérences, de corrections suspectes et de commentaires tendancieux ${ }^{14}$ », alors la condition du philologue, du spécialiste de la lecture et de la textualité, représente, de son point de vue, le paradigme central de l'anthropologie culturelle.

Dans le même temps, la description de Geertz peut être lue comme un commentaire du Rituel du Serpent d'Aby Warburg, ou inversement, cet ouvrage peut apparaître comme une allégorie de la configuration ébauchée par Geertz. Face au Rituel du Serpent nous sommes en effet placés à divers égards dans une situation de lecture. Alors que Warburg s'intéresse, lors du voyage qui le mena auprès des Indiens Pueblos, à leurs symboles, qu'il étudie leurs danses - dans lesquelles il perçoit un «stade [spécifique] de la pensée et de la conduite symboliques ${ }^{15} »-$ ou qu'il décrit les dessins figurant sur leurs poteries à la manière de hiéroglyphes ${ }^{16}$, nous sommes, en tant que lecteurs de ses souvenirs et de ses réflexions théoriques, confrontés dans un premier temps au problème de la lisibilité de son texte. Et dans les correspondances entre sa lecture des Indiens Pueblos et notre lecture de ses écrits, c'est-à-dire entre le problème de la lisibilité de textes culturels et de textes écrits, le concept de lecture subit probablement quelques modifications.

\section{Sur la lisibilité du Rituel du Serpent de Warburg}

Partant des nombreux titres de l'œuvre, une approche archéologique ouvre la perspective sur les différentes strates, ainsi que sur les diverses traductions qui s'y trouvent. Le titre Le Rituel du Serpent provient de la première publication du texte en 1938, qui portait le titre de A Lecture on Serpent Ritual (cette traduction anglaise a été effectuée sur la base de la transcription de la conférence de Kreuzlingen de 1923). Sur la base des esquisses et des brouillons de la conférence, Fritz Saxl et Gertrud Bing, les collaborateurs de Warburg, avaient élaboré une version du texte qui a finalement été publiée pour la première fois en langue allemande sous le titre du Rituel du Serpent. Ainsi ce livre présente-t-il une transcription des notes de Warburg en un texte cohérent. Ceci est tout à fait paradigmatique de la réception de Warburg, dans la mesure où la majeure partie de ses travaux nous a été transmise sous la forme de fragments, de dessins, de blocs-notes, de fichiers et de projets interrompus. Par ailleurs, sa théorie est arrivée jusqu'à nous grâce à ses collaborateurs, ses successeurs et 
héritiers contemporains, c'est-à-dire qu'elle nous a été transmise grâce à des traductions.

S'il est ici question du Rituel du Serpent de Warburg, il ne saurait aucunement s'agir du nom d'un auteur et d'un titre, mais du titre d'un ouvrage dont le texte est contaminé, et ce non seulement dans le sens d'une critique, mais aussi d'une manière comparable à celle des Indiens Pueblos devant lequel le voyageur se tint en 1896 :

«Par ailleurs, la plus grande prudence est requise quand il s'agit de juger les indiens Pueblos du point de vue de la psychologie religieuse, pour une raison bien simple : le matériel est contaminé, c'est-à-dire recouvert d'une double couche. Le fond originel américain est recouvert depuis la fin du XVIe siècle par une couche d'éducation confessionnelle hispano-catholique, dont l'influence s'interrompit brutalement à la fin du XVIIe siècle et reprit par la suite, mais sans pénétrer à nouveau officiellement dans les villages Mokis. Puis vint une troisième couche, celle de l'éducation nord-américaine, qui recouvre le tout ${ }^{17}$. "

Conformément à la référence au problème de la lisibilité qui a été faite précédemment, il ne sera pas question de l'historiographie des couches culturelles nord-américaines mentionnées, mais des strates qui constituent le texte du Rituel du Serpent en tant que tel. Dans cette perspective, la pluralité des titres se révèle être une trace qui, d'un point de vue généalogique, renvoie à la préhistoire du livre. Le genre du "récit de voyage " suggère l'imminence du voyage et le mélange de motifs, tel qu'il ressort des écrits et des lettres de Warburg datant de cette époque, et tel qu'il le rappelle dans ses notes rédigées en vue de la conférence de Kreuzlingen: il s'agit d'une réminiscence romantique de la lecture adolescente de romans d'indiens ${ }^{18}$, de la voie menant à une civilisation "plus naturelle ${ }^{19}$ " que la modernité caractéristique de l'Amérique du Nord ou encore de l'étude de l'art et de la vie d'« un peuple "primitif" qui vient apporter une précieuse rectification à l'étude de toute forme d'art ${ }^{20} »$.

17 Pour autant, le genre du " récit de voyage » a rapidement été relégué au second plan. Alors que Warburg intitule encore «Un voyage à travers le territoire des Indiens Pueblos au Nouveau-Mexique et en Arizona » la première conférence qu'il donna sur ce voyage, huit mois après son retour, devant les membres d'une société de photographes de Hambourg, il la nomma « Images de la vie des Indiens Pueblos » lorsqu'il la présenta peu de temps après à l'association photographique de Berlin ${ }^{21}$. La description d'un voyage se transforma ainsi en une description d'images, contribuant à placer les images au centre. Dans ce contexte, la scène par laquelle Warburg débute, bien des années plus tard, sa conférence à Kreuzlingen dont on connaît le succès, s'annonce déjà : il y parle "d'images avec des mots à l'appui » qui se réfèrent aux "vieux» et "lointains souvenirs » d'un voyage effectué 27 ans auparavant. Lors de la conférence de 1923, il s'agit en effet d'images-souvenirs que vinrent compléter des photographies. Elles sont d'une grande importance puisqu'elles nous rendent attentifs à "l'immédiateté du cliché ». Ces deux formes d'images ont pour but de fournir un portrait de la culture des Indiens sous l'angle des traces mémorielles laissées par les anciennes cultures en Amérique du Nord. Les hésitations de Warburg concernant le titre de sa conférence suggèrent la superposition de ces deux scènes mémorielles. S'il ne parle plus dans le titre de la conférence de Kreuzlingen de la vie, mais du territoire des Indiens Pueblos, il rayera dans les notes prises à ce sujet la formule "souvenirs de voyage" pour la remplacer par "images ${ }^{22}$ ». Il optera finalement pour: "Images du territoire des Indiens Pueblos en Amérique du Nord». Images du territoire...: cette expression 
renvoie à une topographie culturelle derrière laquelle s'efface la scène de ses propres souvenirs et dont il avait fait l'ébauche au début de sa conférence.

Un voyage à travers le territoire..., images de la vie..., images du territoire... : dans ces variations sur le même thème se produisent des décalages inaugurant un mouvement qui s'étend du sujet voyageur à l'objet contemplé, à savoir la vie des Pueblos, avant d'être finalement suspendu et maintenu dans une structure de l'imaginaire: dans les images de ce territoire. La domination des images dissimule et conserve, dans le même temps, la passion qu'avait nourrie une image extraite des archives du romantisme des Indiens, et qui marque pour Warburg l'origine de son voyage :

«Un livre et une image me fournirent le support scientifique ainsi que les objectifs de mon voyage. Ce livre, que je trouvai à la Smithsonian Institution, était l'œuvre de Nordenskjöld sur la "Mesa Verde", ce territoire situé au Nord-Ouest du Colorado où l'on trouve les restes des mystérieux Cliffdwellings - une œuvre solide, portée par un esprit scientifique auquel je dois les fondements de mes tentatives. Le but à la fois romantique et visionnaire qui éveilla chez moi l'envie d'aventure était un imprimé grand format en couleurs, au demeurant de très mauvaise qualité. Il représentait un Indien troglodyte devant un village aménagé dans la faille d'une falaise. C'est lui qui a fait naître mes premières impressions et qui a suscité les questionnements que j'adressai à ses Messieurs de la Smithonian Institution, lesquels me renvoyèrent alors au livre de Nordenskjöld ${ }^{23}$. »

Alors qu'au départ de son voyage, ce livre assura la fonction de "support scientifique " et de «fondement " par rapport à « l'envie d'aventure » éveillée par l'image, l'étude de Warburg, elle, correspond à la tentative de conférer à son analyse d'images un caractère solide parce qu'il s'y consacre en invoquant un "esprit scientifique", l'ouvrant ainsi sur une théorie globale de l'évolution des formes symboliques. Or, cela ne semble possible qu'en éliminant de l'intitulé du sujet les personnages issus des souvenirs de voyage et des images de la vie des Pueblos.

Grâce aux images des territoires pueblos, Warburg veut donner à ses auditeurs de Kreuzlingen "une impression de ce monde qui meurt, en tout cas dans sa culture", tout en posant une question "qui est si décisi[ve] pour toute l'histoire de [notre culture]», à savoir: "Dans quelle mesure devons-nous y voir des caractéristiques essentielles de l'humanité païenne primitive ${ }^{24}$ ?» Ainsi les images des Pueblos sontelles appelées à transmettre une représentation des origines païennes et primitives de la culture européenne qu'il conçoit surtout comme l'histoire du dépassement de la peur par l'intermédiaire de symboles ou, en d'autres termes, comme l'« évolution qui va de l'approche instinctive et magique à l'établissement d'une distance intellectualisante ${ }^{25}$ ». Néanmoins, le sujet, tel qu'il est formulé: "les images du territoire " et le fil conducteur de la conférence, qui est bâtie autour de l'évolution des formes symboliques, ne parviennent qu'imparfaitement à écarter les tensions existant entre les représentations du voyageur européen et les images des Pueblos qu'il analyse.

\section{La notion de symbole et la lecture}

Le Rituel du Serpent montre de manière récurrente comment le problème de la lecture fait irruption dans le projet d'une théorie globale de la formation de symboles. Il y fait littéralement irruption, dans la mesure où les réflexions intervenant à la manière de leitmotivs et portant sur les seuils de formation de symboles sont interrompues par des récits, par des descriptions d'images et de photos souvenirs, qui ne sont pas toujours 
compatibles avec les interprétations symboliques formulées sous forme de concepts. Ainsi Le Rituel du Serpent décrit-il une figure qui est exactement l'inverse de celle du voyage. Car après s'être rendu aux Cliff-Dwellings et chez les Indiens Pueblos du Nouveau-Mexique, Warburg avait interrompu son voyage pour un assez long séjour dans les universités de Californie avant de se rendre chez les Pueblos d'Arizona. Durant ce séjour de recherches, il avait pris des notes concernant un système global de formes symboliques qui devait ensuite faire office pour lui de "fondement» ou de support scientifique pour l'analyse d'autres images : «Je crois que j'ai enfin trouvé l'expression qui s'applique à ma loi psychologique ; je la cherchais depuis $1888^{26}$. "

21 L'année 1888 marque le début de sa thèse de doctorat sur Botticelli. C'est cette thèse qui est à l'origine de ses interrogations relatives à l'importance des "accessoires en mouvement " et des gestes d'émotion sur les images. Enfin, cette année-là marque également le début du transfert de sa sphère d'intérêts de l'histoire de l'art vers la psychologie. Aussi le thème de la survivance de l'Antiquité dans l'art renaissant annonce-t-il déjà la " "psychologie historique de l'expression humaine" qu'il reste à écrire $^{27}$ ». La phrase précédemment citée, qu'il a notée durant l'interruption de son voyage et dans laquelle il parle de "la loi psychologique " trouvée en observant les «actions religieuses des Indiens Pueblos» situe la visite de Warburg chez les Pueblos dans la généalogie de ses propres découvertes et de sa propre topographie, lui attribuant ainsi le caractère d'une révélation. Cette halte durant le voyage et la découverte d'une telle loi créent un lien entre l'expérience ethnologique et les études de l'art renaissant, conférant également au voyage l'aspect d'un « rite de passage ${ }^{28} » . \mathrm{Au}$ terme de ce dernier, le voyageur dispose d'une nomenclature qui va lui permettre de s'orienter dans la découverte d'autres territoires, lesquels seront toutefois situés à nouveau sur le territoire européen.

La découverte de cette loi, dont le nom subira plusieurs modifications dans les études ultérieures («l'expression de ma loi psychologique » devenant en effet la " psychologie de l'expression »), fait office de principe organisationnel et de pierre angulaire au vu de l'extension du champ de ses découvertes en matière d'histoire de l'art en Europe. Ceci ne fait aucun doute si l'on considère l'augmentation du fonds de sa bibliothèque. La fascination qu'il éprouve pour sa matière et pour les variations de détails, la manière dont les études de Warburg se perdent dans les archives les plus diverses ainsi que dans les livres, les notes et les reproductions d'images qui s'amoncellent autour de lui forment un étrange contraste avec les efforts terminologiques et théoriques qu'il fournit lorsqu'il travaille, en cultivant les stéréotypes, à la formulation d'une loi concernant la construction de symboles humains.

Dès lors que la conception du symbole selon Warburg devient notionnelle, c'est tout le poids de la pensée biologique et évolutionniste représentative du XIXe siècle qui se fait sentir, que ce soit au travers de la description d'une évolution menant de la pulsion (du corps vers l'esprit, de la magie vers la raison, de l'obscurité vers la lumière) ou au sujet de la civilisation, perçue comme un travail de dédiabolisation et un processus de purification. Aux origines de l'histoire se trouve selon lui une phobie dont on vient à bout en traversant les étapes des formes magique, mythique, symbolique et logique. Plus précisément, en rétablissant toujours et encore la distance qui sépare l'Homme d'une nature menaçante. Dans l'accroissement de cette distance, Warburg situe la naissance d'un « espace de pensée » [Denkraum]. Ce paradigme ressortissant à la théorie de l'évolution est également inscrit dans Le Rituel du Serpent : 
"L'évolution de la culture vers l'ère de la raison se mesure à la manière dont le foisonnement tangible et frustre de la vie s'estompe pour devenir un signe mathématique ${ }^{29}$.»

«La pensée mythique et la pensée symbolique, en luttant pour donner une dimension spirituelle à la relation de l'homme à son environnement, ont fait de l'espace une zone de contemplation ou de pensée, espace que la communication électrique instantanée anéantit ${ }^{30}$. »

Partant des modèles de la mémoire organique développés par Richard Semon et Ewald Hering, ses réflexions d'ordre théorique sur la mémoire sociale ont aussi certains fondements biologiques. Elles annoncent de surcroît la reformulation de l'inconscient à travers la théorie du signe, telle qu'elle a été entreprise par la psychanalyse freudienne. C'est surtout dans le discours de Warburg sur la bordure de frappe, sur «l'inscription dans l'âme de valeurs expressives prédéterminées", sur les «engrammes de l'expérience de la passion » que le corps apparaît comme l'empreinte de processus d'émotions psychiques. On renverra à ce sujet à l'Introduction à l'Atlas Mnémosyne de $1929^{31}$.Dans ce sillage, les «formules du pathos » constituent rapidement un répertoire de formules iconographiques figées, les symboles acquièrent une signification codée ou décodable, pouvant ainsi être facilement intégrés à un modèle iconographique traditionnel, comme cela se présente pour la majeure partie de la réception de Warburg.

Cependant, dans un tel répertoire s'estompent les traces de la curiosité, qui sont caractéristiques du travail de Warburg et qui laissent entrevoir une lecture des symboles parfaitement opposée. Dans la lecture d'images isolées, l'expression gestuelle est par exemple conçue comme une forme symbolique qui ne peut être traduite par le langage, parce qu'elle ne peut être déchiffrée que comme un symptôme, c'est-à-dire comme le symbole mémoriel d'émotions passées. Dans l'Atlas d'images Mnémosyne, dans le projet consistant à classer par groupes les «formules du pathos » et les formules imagées, on peut voir sur les planches créées par Warburg des séries d'images qui doivent être conçues comme des traces mémorielles à l'intérieur desquelles chaque répétition décrit une déviance et chaque image d'une série revient sous une forme altérée.

Dans cette mesure, il me paraît bien plus fructueux d'analyser les traces laissées par la curiosité de Warburg, ainsi que d'étudier les voies qu'il a empruntées pour procéder à ses lectures que de tenter de présenter ses travaux sous la forme d'un édifice cohérent. Prenons l'exemple de la bibliothèque de Warburg : ainsi que Kurt W. Forster l'a montré, elle fait figure de représentation matérielle de l'édifice que constitue sa pensée, présentant des liens avec les cultes dont Warburg a étudié la pratique ${ }^{32}$, mais son organisation va totalement à l'encontre de l'ordre établi en matière d'archivage, ce qui avait déjà conduit son collaborateur Saxl à vouloir la "normaliser », i. e. la " simplifier"33 ». La réaction d'Ernst Cassirer devant cette bibliothèque, affirmant qu'il devait soit l'éviter soit s'y enfermer durant plusieurs années ${ }^{34}$, laisse penser que l'on retrouve précisément dans les traces matérialisées des études de Warburg cette figure dont il a fait un objet de travail. Tandis que la loi qu'il a découverte décrit le dépassement des origines sauvages, on perçoit en effet, disséminés dans les traces de son travail, dans sa fascination pour la matière, les signes d'un état sauvage.

On a volontiers mis en relation l'interprétation de l'Histoire et l'introspection chez Warburg, sa maladie ayant donné l'occasion d'établir, comme le souligne son médecin Ludwig Binswanger, « des points de passage entre ses vues scientifiques et la folie de 
ses idées ${ }^{35}$ ». Or, on pourra objecter qu'une telle interprétation dissimule, au nom d'une pathologie, les correspondances existant entre l'objet d'étude et la méthodologie de travail. Lorsque Warburg dit de sa situation à Kreuzlingen qu'elle est un «enfer » et qu'il veut faire de sa conférence la preuve de sa bonne santé, il l'envisage selon le même schéma que celui avec lequel il avait inscrit les images des Pueblos dans une histoire de la symbolisation. Le travail et la pensée de Warburg, qui sont constitués de moments de lutte contre la phobie et de schémas passionnels, et qui recherchent une sorte de salut dans la découverte de lois générales, correspondent exactement à la théorie des symboles qu'il a développée, à la différence cependant qu'ils permettent de déceler les tensions qui se cristallisent dans le concept de symbole.

\section{Sur le motif du retour dans Le Rituel du Serpent de Warburg}

Cela vaut tout autant pour Le Rituel du Serpent de Warburg. Dans cette mesure, la notion de symbole qu'il y forge me semble aujourd'hui moins intéressante. Les références les plus variées y ont de surcroît été reliées : Vignoli, Durkheim, la théorie de la forme du concept dans la pensée mythique selon Cassirer ${ }^{36}$, la signification des rituels en tant que forme de culture préexistante aux mythes - telle qu'elle a été mise en exergue par l'école ritualiste de Cambridge autour de Frazer, les restes d'un modèle héréditaire biologique et, enfin, le motif ontologique de l'ascension que l'on doit probablement à des entretiens avec Binswanger ${ }^{37}$.

En revanche, la lecture mêlant, d'une part, le problème de la lisibilité des images tirées des territoires des Indiens Pueblos et, d'autre part, celui de la lisibilité du texte de Warburg me paraît bien plus édifiante. La devise qui se trouve en exergue de la conférence nous place devant une énigme, tout en nous fournissant certains indices :

"C'est un vieux livre à feuilleter,

Athènes-Oraibi, rien que des cousins ${ }^{38}$."

Cette formule contient une double citation. En tant que lien intertextuel crypté et modifié, il met en relation la conférence de Kreuzlingen avec le dernier ouvrage que Warburg avait achevé avant sa maladie, à savoir l'étude consacrée à «La divination païenne et antique dans les écrits et les images à l'époque de Luther » (1920). De cette façon, ce motif jette un pont entre les images des Pueblos et le thème de la simultanéité de la logique et de la magie à l'époque de la Réforme en Europe :

«C'est un vieux livre à feuilleter :

Du Harz à l'Hellas, toujours des cousins.

Faust II ${ }^{39}$ »

Cette citation attire par conséquent l'attention sur une constellation d'images qui, de la même manière que le voyage de 1896 et l'étude sur Botticelli de 1888, met en lien la conférence sur les Indiens Pueblos et les images de l'époque luthérienne, si bien que la conférence de Kreuzlingen peut être perçue comme la répétition d'un «rite de passage $»^{40}$. La tournure concernant la parenté des lieux, «toujours", (à savoir "rien que des cousins »), rejoint la problématique générale énoncée dans Le Rituel du Serpent :

«[...] dans quelle mesure la vision païenne du monde, telle qu'elle existe encore chez les Indiens pueblos, nous fournit-elle un critère de l'évolution qui conduit du primitif païen à l'homme moderne, en passant par l'homme païen de l'Antiquité classique ${ }^{41} ? »$ 
Elle se rattache aussi aux notes prises en vue de la conférence de Kreuzlingen, dans lesquelles Warburg parle d'une "recherche comparée de l'éternelle essence indienne dans une âme humaine désemparée $»^{42}$.

Néanmoins, cette parenté se complexifie lorsque l'on s'attache à la topographie concrète des lieux. Alors que malgré la transformation de la citation, les noms Hellas et Athènes forment une constante, Oraibi apparaît face à la chaîne montagneuse du Harz. Ainsi un parallèle est-il établi entre le voyage chez les Pueblos, c'est-à-dire chez les Indiens, et le voyage du Faust de Goethe dans le Harz. Cependant, la direction a été inversée au cours de cette substitution: "Du Harz à l'Hellas » devient en effet un voyage en sens inverse « Athène-Oraibi ».

Dans l'original, le Faust II de Goethe, les paroles prononcées par Méphistophélès sont précédées des lignes suivantes: «je pensais ne voir ici que des inconnus, / Et je ne trouve, hélas ! que de proches parents » (7740-1). Il est en somme question du rapport qu'entretiennent le connu et l'inconnu, sachant que ce rapport se présente à Méphisto dans le scénario mysthico-cosmique de la "nuit classique de Walpurgis ", peuplée de créatures issues du monde démoniaque des Dieux antiques, de telle sorte que les sorcières qu'il connait du mont Blocksberg lui apparaissent comme les connaissances les plus inoffensives :

"Les sorcières nordiques, je savais bien m'en rendre maître,/ Mais je ne me sens pas à l'aise avec ces esprits étrangers./ Le Blocksberg reste un endroit bien confortable,/ Où que l'on soit, on se retrouve toujours. » (7677-9)

«Je me serais pas imaginé pareille extravagance,/ Une telle montagne, en une seule nuit./ J'appelle cela une fort leste chevauchée de sorcières:/ Elles apportent leur Blocksberg avec elles. » (7807-10)

Si, dans l'étude sur Luther, les esprits de l'Antiquité de la Nuit classique de Walpurgis représentent pour Warburg l'inconnu et si l'expression en est la présence d'éléments "païens et antiques » durant l'époque de la Réforme, alors l'inversion de l'orientation perceptible dans la citation "Athène-Oraibi» du Rituel du Serpent, suggère une inversion du rapport entre l'inconnu et le connu qui est inscrite dans les images des Pueblos. Or, on observe ici en même temps un décalage. Si dans la problématique générale du Rituel du Serpent, la survivance chez les Pueblos d'une "conception païenne » a été introduite en tant que critère d'une évolution douteuse, ce critère semble disparaître à mesure que s'accentue le problème de la lisibilité des images des Pueblos. Si nous ne considérons pas la formule « Athène-Oraibi », à l'image de celle « du Harz à l'Hellas ", comme la direction d'un voyage, mais comme l'indication d'un lieu donné, alors celle-ci marque dans Le Rituel du Serpent une sorte de point de renversement.

31 Après la description des habitations des Pueblos, des peintures figurant sur leurs poteries, de leur représentation cosmologique du monde et de la «danse des antilopes » - une imitation animale qui peut être interprétée comme "la recherche de moyens de subsistance par le groupe social grâce à des pratiques magiques ${ }^{43}$, Oraibi apparaît comme «l'endroit situé le plus à l'Ouest $»^{44}$. À Oraibi, la dernière étape de son voyage, Warburg put en effet observer la danse "Humis-katcina", une danse de masques qu'il associe à un culte magique, mais dont il ne comprend pas toute la signification. Il se peut que la superposition du motif du spectacle à la description de ce rituel soit due au trouble que génère le rapport entre l'imitation et l'original : «c'est là que j'ai pu voir les originaux vivants des danseurs de masques que j'avais vus auparavant dans une pièce de ce même village d'Oraibi sous forme de poupées $[. ..] »^{45}$. 
Mais si les poupées sont les imitations de masques, de quoi les masques sont-ils donc les imitations?

Afin de mettre un peu d'ordre dans ce spectacle surprenant, le texte s'appuie sur un savoir que tout le monde possède, la tragédie antique : " pour peu que l'on connaisse un peu la tragédie antique, on retrouvera ici $[. ..] »^{46}$. Après un passage sur « la danse avec les serpents vivants $\aleph^{47}$, le texte abandonne les images des territoires pueblos, les remplaçant par des images de l'Antiquité. Si "Athènes-Oraibi » caractérise précisément, dans la citation placée en exergue du texte, ce passage précis dans lequel les archives iconographiques changent, la direction demeure pourtant inversée : elle conduit en effet d'Oraibi à Athènes. Elle ne conduit pas - comme pour le Harz jusqu'à l'Hellas - du connu vers l'inconnu, mais, à l'inverse, de l'inconnu vers le connu, vers l'Antiquité qui est profondément ancrée dans la mémoire iconographique européenne. Ainsi la perception d'une culture étrangère est-elle représentée sous les traits d'une culture certes passée, mais de toute évidence familière.

On notera en outre que ce passage est organisé autour d'un espace exempt d'images. Il concerne le rituel qui donne son titre à l'ouvrage :

«L'apogée de cette tentative magique d'approcher la nature à travers le monde animal s'observe à Oraibi et à Walpi chez les Indiens mokis [...], dans la danse avec les serpents vivants. Je n'ai pas observé cette danse moi-même, mais quelques photographies donnent une idée de la cérémonie, la plus païenne de toutes, à Walpi ${ }^{48}$."

Là où le nom d'Oraibi indique la fin du voyage de Warburg dans une culture étrangère, commence le voyage dans son propre imaginaire : celui-ci porte le nom d'Athènes. Là où se perdent ses images d'une culture étrangère, elles sont remplacées par des images extraites de la mémoire visuelle de l'histoire de la culture européenne. On ne peut donc pas affirmer, à l'instar de Peter Burke, que le détour effectué par Warburg auprès d'autres cultures lui a permis de mieux comprendre l'Antiquité et la Renaissance. Les traces que laisse la lecture du Rituel du Serpent esquissent un chemin inverse, voire plusieurs changements de direction. La citation, qui va à l'encontre du mouvement suivi par le texte et qui est parallèlement une inversion du motif emprunté à Goethe, renverse le rapport entre le connu et l'inconnu. Il peut être lu comme un signe indiquant ce double retour dans la lecture des images tirées des territoires pueblos. Par conséquent, il semble possible d'interpréter l'histoire que relate Le Rituel du Serpent non plus sous l'angle d'une histoire de l'évolution symbolique. Mais il s'agit là encore d'une question de lecture.

\section{BIBLIOGRAPHIE}

Barta Fliedl, I. / Geissamann, C. (éd.) (1992) : Die Beredsamkeit des Leibes. Zur Körpersprache in der Kunst, Salzbourg / Vienne : Residenz Verlag.

Baumgart, S. et. al. (éd.) (1993) : Denkräume zwischen Kunst und Wissenschaft. 5.

Kunsthistorikerinnentagung in Hamburg, Berlin : Reimer. 
Benjamin, W. (1979) : « Johann Jacob Bachofen », Text und Kritik : Benjamin, 31/32, p. 28-40.

Benjamin, W. (1980) : Gesammelte Schriften, Francfort-sur-le-Main : Suhrkamp.

Benjamin, W. (1985) : Origine du drame baroque allemand, trad. de l'allemand par S. Muller (avec le concours de A. Hirt), Paris : Flammarion.

Binswanger, L. (1954) : Le Rêve et l'Existence, trad. de l'allemand par J. Verdeaux, introduction et notes de M. Foucault, Paris : Desclée.

Burke, P. (1991) : « Aby Warburg as Historical Anthropologist. », in : Bredekamp, H. / Diers, M. / Schoell-Glass, Ch. (1991), p. 39-44.

Burkert, W. (1990) :Wilder Ursprung. Opferritual und Mythos bei den Griechen, Berlin : Wagenbach.

Cassirer, E. (1997) : « La forme du concept dans la pensée mythique », in : id. : Trois essais sur le symbolique, trad. de l'allemand par J. Carro (avec la collaboration de J. Gaubert), Paris : Les Éditions du Cerf, p. 39-111.

Diers, M. (1979) : « Kreuzlinger Passion », Kritische Berichte, 4/5, p. 5-14.

Diers, M. (1991) : Warburg aus den Briefen. Kommentare zu den Kopierbüchern der Jahre 1905-1918, Weinheim :VCH Acta Humaniora.

Forster, Kurt W. : « Die Hamburg-Amerika-Linie, oder : Warburgs Kulturwissenschaft zwischen den Kontinenten », in : Hort Bredekamp, Michael, Diers et Charlotte Schoell-Glass (éds.) : Aby Warburg. Akten des internationalen Symposiums Hamburg 1990, Weinheim, VCH Acta Humaniora 1991, p. 11-37.

Füssel, S. (éd.) (1979) : Mnemosyne. Beiträge zum 50. Todestag von Aby M. Warburg, Göttingen : Gratia Geertz, C. (1998) : « La description dense », trad. de l'anglais par A. Mary, Enquête : La description I, [En ligne], mis en ligne le 27 janvier 2009. URL : http://enquete.revues.org/ document1443.html, consulté le 21/03/2012.

Goethe, J. W. von (2009) : Faust. Urfaut, Faust I, Faust II, édition établie, présentée et annotée par J. Lacoste et J. Le Rider, Paris :Bartillat.

Gombrich, E. H. (1970) : Aby Warburg. Eine intellektuelle Biographie, Hambourg : Europäische Verlagsanstalt.

Hering, E. (1921) : Über das Gedächtnis als allgemeine Funktion der organisierten Materie, Leipzig : Akademische Verlagsgesellschaft.

Hofmann, W. / Syamken, G. / Warnke, M. (1980) : Die Menschenrechte des Auges. Über Aby Warburg, Francfort-sur-le-Main : Europäische Verlagsanstalt.

Janshen, F. (1993) : « Spurenlesen. Um Aby Warburgs ‘Schlangenritual’ », Denkräume, p. 86-112.

Kaemmerling, E. (1979) : Ikonographie und Ikonologie. Theorie, Entwicklung, Probleme, Cologne : DuMont.

Liebeschütz, H. (1971) : « Aby Warburg (1866-1929) as Interpreter of Civilisation », in : Leo Beck Institute Yearbook, 16, London / Jerusalem / New York, p. 225-236.

Naber, C. (1988) : « Pompeji in Neu-Mexiko. Aby Warburgs amerikanische Reise », Freibeuter, 38, p. 88-97.

Pochat, G. (1983) : Der Symbolbegriff in der Ästhetik und Kunstwissenschaft, Cologne : DuMont.

Raulff, U. (1991) : « Zur Korrespondenz Ludwig Binswanger-Aby Warburg im Universitätsarchiv Tübingen. », in : Bredekamp, H. / Diers, M. / Schoell-Glass, Ch. (1991), p. 55-70. 
Saxl, F. (1980) : « Warburgs Besuch in Neu-Mexiko », in : id. : Warburg, p. 317-326.

Schlesier, R. (n. p.) : « “Arbeiter in Useners Weinberg”. Anthropologie und antike Religionsgeschichte in Deutschland nach dem 1. Weltkrieg. ", manuscript non publié.

Semon, R. (1980) : Die Mneme als erhaltendes Prinzip im Wechsel des organischen Lebens, Leipzig :

W. Engelmann.

Settis, S. (1991) : « Empfehlungen für eine Heimkehr », in : Bredekamp, H. / Diers, M. / SchoellGlass, Ch. (éd.) (1991) : Aby Warburg. Akten des internationalen Symposiums Hamburg 1990, Weinheim : VCH Acta Humania, p. 115-123.

Warburg, A. (1932) : Gesammelte Schriften, Leipzig / Berlin : B. G. Teubner.

Warburg, A. (1980) : Ausgewählte Schriften und Würdigungen, Baden-Baden : V. Koerner.

Warburg, A. (1990) : Essais Florentins, trad. de l'allemand par S. Muller, Paris : Editions Klincksieck.

Warburg, A. (1993) : Bildersammlung zur Geschichte von Sternglaube und Sternkunde im Hamburger Planetarium, Hambourg : Dölling und Galitz.

Warburg, A. (1993a) : Mnemosyme-Atlas. Begleittext zur Ausstellung, Akademie der Künste Wien, Vienne : Daedalus.

Warburg, A. (1994) : «Introduction à l'Atlas Mnémosyne », trad. de l'allemand par P. Rusch, Trafic, 9.

Warburg, A. (2003) : Le Rituel du serpent. Récit d'un voyage en pays pueblo, trad. de l'allemand par S. Muller et Ph. Guiton; de l'italien par D. H. Bodart, Paris : Macula.

\section{NOTES}

1. Cette contribution est la conférence inaugurale que j'ai donnée à l'Université de Zurich le $1^{\mathrm{er}}$ novembre 1993.

Mon poste au Département d'Allemand de l'Université de Zurich correspondait pour moi - après que j'ai effectué un détour en participant durant plusieurs années à la création de l'Institut des Sciences de la Culture [Kulturwissenschaftliches Institut] en Rhénanie du Nord-Westphalie - à une sorte de retour vers la discipline dont je suis issue : les études littéraires. Un tel retour ne menant jamais vers le même lieu, mais toujours vers un autre, cette conférence inaugurale fut pour moi l'occasion d'ouvrir une réflexion sur la figure du retour, c'est-à-dire de partir du point de vue des études littéraires pour éprouver, tout en se retournant, la validité de perspectives propres aux sciences de la culture. Comme le dit Walter Benjamin : «[1]a méthode est détour »; Benjamin (1985), p. 24.

2. Burkert (1990).

3. Cf. Warburg (1993), (1993a).

4. Janvier/février 1896 au Nouveau Mexique, avril/mai 1986 en Arizona ; pour le parcours exact du voyage cf. Naber (1988).

5. Burke (1991), p. 44.

6. À ce sujet, cf. l'essai de Warburg sur les fresques du Palazzo de Schifanoia à Ferrare : Warburg (1990), p. 215.

7. Benjamin (1979), p. 32. À ce sujet, cf. l'hommage que Benjamin rend à Warburg dans la recension « Strenge Kunstwissenschaft » de 1931 : Benjamin (1980), vol. 3, p. 374 ; cf. également le renvoi à Warburg et à l'école de Warburg dans l'ouvrage sur le théâtre baroque : Benjamin (1985), p. $162,239$. 
8. Settis (1991), p. 122.

9. Ibid.

10. Schlesier 1993, 13.

11. Warburg (1932), t. II, p. 479.

12. Ceci est explicite dans les "notes de la conférence» de Kreuzlingen. Cf. à ce propos: Gombrich (1992), p. 295 sqq.

13. Pour un renvoi à Gilbert Ryle cf. Geertz (1998).

14. Geertz (1998).

15. Warburg (2003), p. 77.

16. Warburg (2003), p. 66.

17. Warburg (2003), p. 60.

18. Notes rédigées en vue de la conférence, citées d'après Gombrich (1992), p. 36 sq.

19. Ibid., p. 117.

20. Lettre de 1895, citée d'après Naber (1988), p. 95.

21. Cf. Naber (1988), p. 96.

22. Cf. Janshen (1993), p. 104. Janshen s'est tout d'abord attaché au problème de la textualité et de la scène d'écriture du Rituel du Serpent, en les traitant du point de vue de l'auteur, de l'œuvre et des citations et non de celui de la lisibilité et du contexte ethnographique.

23. Notices rédigées en vue de la conférence. Citées d'après Naber (1988), p. 89 sq.

24. Warburg (2003), p. 59.

25. Warburg (2003), p. 131.

26. Notes prises durant le voyage ; citées d'après Gombrich (1992), p. 121.

27. Warburg (1933), t. II, p. 478.

28. En français dans le texte [NdT].

29. Warburg (2003), p. 120.

30. Warburg (2003), p. 133.

31. Warburg (1994), p. 41 sq.

32. Forster (1991), p. 18 sq.

33. Saxl (1980), p. 341.

34. Saxl (1980), p. 340.

35. Pour un point de vue critique; cf. Diers (1979).

36. Cf. Cassirer (1997).

37. Cf. Binswanger (1954), p. 80 sqq.

38. Warburg (2003), p. 57.

39. Warburg (1980), p. 201.

40. En français dans le texte [NdT].

41. Warburg (2003), p. 61.

42. Cité d'après Gombrich (1992), p. 304.

43. Warburg (2003), p. 74.

44. Warburg (2003), p. 83.

45. Ibid.

46. Warburg (2003), p. 101.

47. Warburg (2003), p. 103.

48. Ibid. 
INDEX

Mots-clés : texte culturel, anthropologie culturelle, lisibilité

Schlüsselwörter : kultureller Text, Kulturanthropologie, Lesbarkeit

\section{AUTEURS}

\section{SIGRID WEIGEL}

Sigrid Weigel est professeur au département de littérature à l'Université Technique de Berlin. Pour plus d'informations, voir la notice suivante. 